\title{
A possible explanation for cricoid pressure to improve the laryngeal view with the Truview Evo2 ${ }^{\mathrm{TM}}$ laryngoscope
}

\author{
Fu S. Xue, MD • Yu J. Yuan, MD • \\ Jian H. Liu, MD
}

Received: 25 July 2011/ Accepted: 14 September 2011/Published online: 5 October 2011

(C) Canadian Anesthesiologists' Society 2011

\section{To the Editor,}

In a randomized clinical trial, Kumar et al. ${ }^{1}$ showed that the application of cricoid pressure (CP) improved the laryngeal view with a Truview Evo $2^{\mathrm{TM}}$ laryngoscope (TEL) without altering the ease of tracheal intubation. However, the authors did not specify the size of blade used in their study. The TEL blade is available in two adult sizes with lengths of $129 \mathrm{~mm}$ and $143 \mathrm{~mm}$, and proper functioning of the TEL may depend on using a blade of an appropriate length. Similarly, the authors made no mention of an attempt to standardize the lifting force of the laryngoscope. Increased lifting force can alter the resultant laryngeal view, and this increase can occur in response to a poor view without the laryngoscopist even being aware. ${ }^{2} \mathrm{We}$ suggest an alternative explanation why $\mathrm{CP}$ can improve the laryngeal view with the TEL.

In patients with normal and difficult airways, the TEL has been shown to provide a better laryngeal view than the Macintosh laryngoscope due to its tubular optical system that provides an unmagnified laryngeal view at a $42^{\circ}$ anterior refracted angle. ${ }^{3,4}$ However, the manufacturer's description specifies that the upper refracted line of sight of the device's optical system passes just below the blade tip (www.truphatek.com) (Figure, Panel A). That is, during tracheal intubation with the TEL, the blade tip can always be seen. Actually, the visual field obtained by the tubular

F. S. Xue, MD $(\varangle)$ · Y. J. Yuan, MD · J. H. Liu, MD Plastic Surgery Hospital, Chinese Academy of Medical Sciences and Peking Union Medical College, Beijing,

People's Republic of China

e-mail: fruitxue@yahoo.com.cn optical system of the device cannot cover the blade tip, resulting in a blind area below the blade tip. Measurements, as shown in the Figure 1 (Panel B), show that the blind area below the blade tip of the device's tubular optical system is $2,4,7,9$, and $11 \mathrm{~mm}$ in size $0,1,2,3$, and 4 blades, respectively. This makes it difficult to visualize what is happening at the blade tip during tracheal intubation, perhaps because there is a relatively large anterior curve between the distal portion and the tip of the blade. Furthermore, a similar flaw has been found in the GlideScope ${ }^{\circledR}$ videolaryngoscope, which also has an accentuated curve on its blade. 5

The visual field obtained by the tubular optical system of the TEL includes only the glottis without the surroundings. The length of the adult glottis is about $23 \mathrm{~mm}$ in males and $17 \mathrm{~mm}$ in females. Thus, when an adult blade (size 3 or 4 ) is used, a 9-11 mm blind spot below the blade tip can undoubtedly interfere with the laryngeal exposure with the device (Figure 1, Panel C). Moreover, this issue may be especially significant in patients with their larynx in a more anterior position, as it requires a visual field in a more anterior direction. ${ }^{5}$ Nevertheless, when the external force of the $\mathrm{CP}$ results in a downward movement of the larynx, the upper part of the glottis, which cannot be seen, will be moved out of the blind spot, and the TEL will provide an improved laryngeal view. With a gentle lifting force of the TEL, ${ }^{1}$ perhaps the favourable effect of the CP on the laryngeal view will be more apparent. In practice, both external laryngeal pressure and CP have previously been used with this device as optimization maneuvers to aid laryngeal exposure and tracheal intubation in adult patients. ${ }^{4}$ In summary, the TEL appears to have a blind spot below its blade tip, and external laryngeal pressure or $\mathrm{CP}$ may be used with this device to improve the laryngeal view during laryngoscopy. 

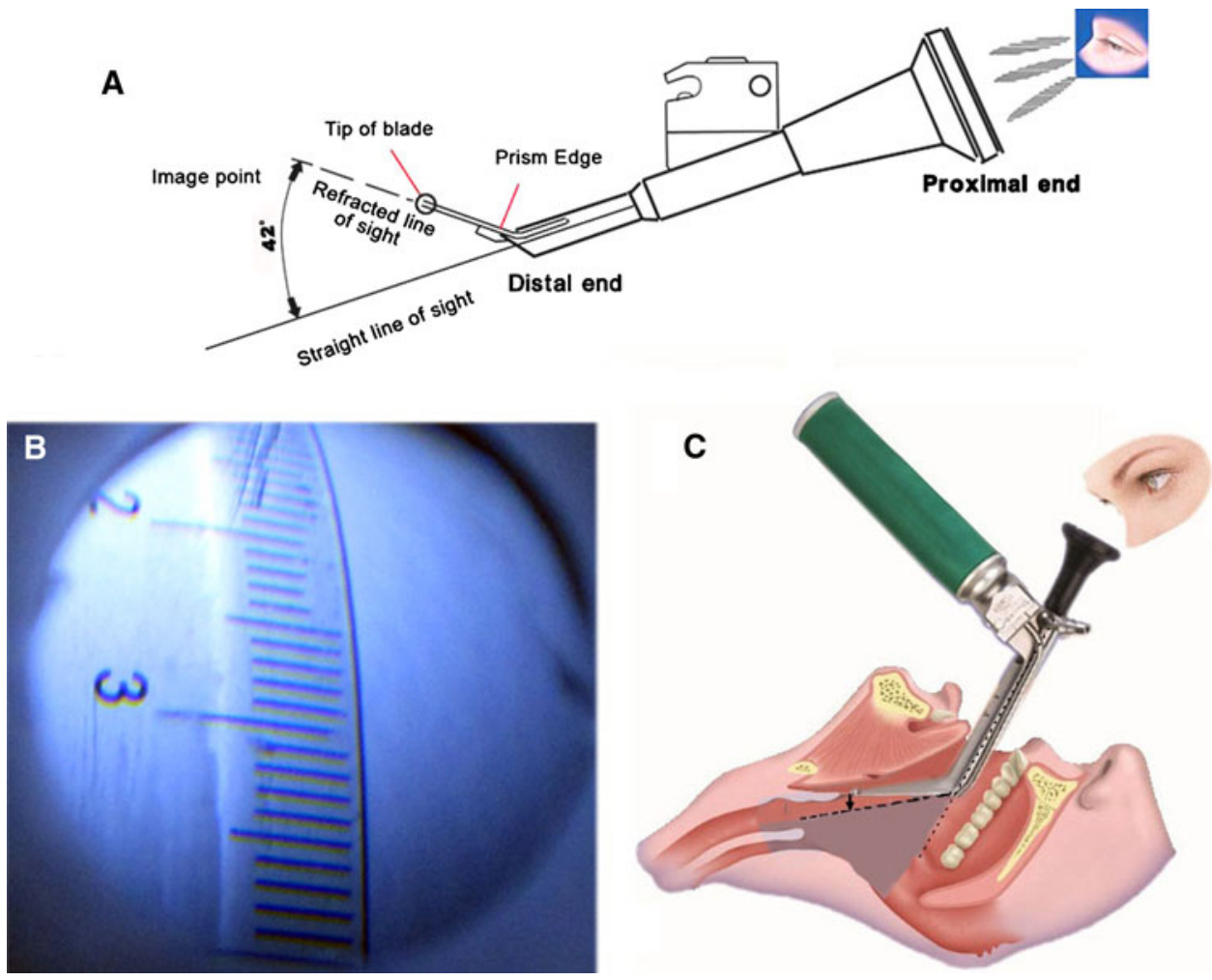

Figure In the manufacturer's description, the upper refracted line of sight of the tubular optical system of the TruView Evo $2^{\mathrm{TM}}$ laryngoscope is described to pass just below the distal tip of the blade (Panel A). Panel B represents a photographic image of a ruler obtained through a size 4 blade of the TruView Evo $2^{\mathrm{TM}}$ laryngoscope. The ruler was placed perpendicular to the direction of the blade tip with the blade tip placed at $0 \mathrm{~mm}$ on the ruler's scale. The blade shows graduations from $12 \mathrm{~mm}$ and above. Thus, there is an 11-mm

Competing interests None declared.

\section{References}

1. Kumar N, Behera D, Dali JS, Arya M, Gupta A. Cricoid pressure with the Truview Evo2 ${ }^{\mathrm{TM}}$ laryngoscope improves the glottic view. Can J Anesth 2011; 58: 810-4.

2. Hastings RH, Hon ED, Nghiem C, Wahrenbrock EA. Force and torque vary between laryngoscopists and laryngoscope blades. Anesth Analg 1996; 82: 462-8.

3. Li JB, Xiong YC, Wang XL, et al. An evaluation of the TruView EVO2 laryngoscope. Anaesthesia 2007; 62: 940-3.

4. Malik MA, Maharaj CH, Harte BH, Laffey JG. Comparison of Macintosh, Truview ${ }^{\circledR}$ EVO2, Glidescope ${ }^{\circledR}$, and Airwayscope ${ }^{\circledR}$ laryngoscope use in patients with cervical spine immobilization. Br J Anaesth 2008; 101: 723-30.

5. Hirabayashi Y, Otsuka Y. Apparent blind spot with the GlideScope $^{\circledR}$ video laryngoscope. Br J Anaesth 2009; 103: 461-2.

\section{Reply,}

We thank Drs. Xue et al. for their interest in our trial. They have proposed an interesting hypothesis regarding the

blind area at the top of the camera's screen. Panel C shows a correctly simulated image of laryngeal exposure with the TruView Evo $2^{\mathrm{TM}}$ laryngoscope. In this panel, the shadow denotes the field of view with the TruView Evo2 ${ }^{\mathrm{TM}}$ laryngoscope, and the black arrow shows the size of the blind area below the blade tip. Panels $\mathrm{A}$ and $\mathrm{C}$ are modified from the website of TruView Evo2 ${ }^{\mathrm{TM}}$ laryngoscope with the manufacturer's permission (www.truphatek.com)

possible reason for improvement of the glottic view with the application of cricoid pressure (CP) using the Truview Evo $2^{\mathrm{TM}}$ laryngoscope.

We used a size 3 adult blade $(129 \mathrm{~mm})$ in all patients during our trial. There is no doubt that the degree of force used for laryngoscopy varies between laryngoscopists and laryngoscope blades, but patient factors also play a major role. Each patient will require a different force for the best glottic view, even in the hands of the same laryngoscopist and laryngoscope blade. Thus, standardizing the degree of force applied would not have resulted in the best possible glottic exposure in all patients. In fact, we have mentioned the inability to measure the lifting force as one of the limitations of the study. However, the downward force applied (CP) was standardized to $40 \mathrm{~N}$.

We agree with the authors regarding the blind spot obscuring the view, but only in principle. We can approach the problem in another way: Had the blind spot been 9-11 mm with sizes 3 and 4 blades, respectively, almost $50 \%$ of the glottis would remain hidden from view. We obtained a mean glottis opening (POGO) score of $81 \%$ 
without the application of $\mathrm{CP} .{ }^{1}$ Assuming the mean anteroposterior diameter of the adult larynx is $22 \mathrm{~mm}$ and the blind spot is $9 \mathrm{~mm}$, the POGO score would be only $60 \%$, which is in contrast to the scores reported by us ${ }^{1,2}$ and others ${ }^{3-5}$ in previous studies! The authors failed to take into account that the tip of the blade is placed in the vallecula. The blind spot may cover the thickness of soft tissue between the vallecula and the anterior commissure, thus not affecting the glottic view at all! If this had not been the case, a $100 \%$ POGO score would not be possible with any videolaryngoscope, thus mitigating their utility.

In fact, observations made by us in a similar fashion have shown that the blind spot with a size 3 blade was approximately $5 \mathrm{~mm}$, which is clinically insignificant. Also, altering the angulation of the blade to the horizontal plane altered the width of the blind spot. Although the in vitro observations seem appealing, actual photographic measurements of the blind spot and glottic view are required with the videolaryngoscopes in situ before we can satisfactorily implicate the blind spot to poor views of the glottis.

Financial support None.

Conflict of interest None declared.

\section{References}

1. Kumar N, Behera D, Dali JS, Arya M, Gupta A. Cricoid pressure with the Truview Evo2 ${ }^{\mathrm{TM}}$ laryngoscope improves the glottic view. Can J Anesth 2011; 58: 810-4.

2. Kumar N, Lahkar D, Kumar A, Lamba K, Ratra M. A randomised study comparing Truview Evo2 laryngoscope with and without the use of camera. J Anaesthesiol Clin Pharmacol 2010; 26: 365-70.

3. Bharti N, Arora V, Wig J. TruView laryngoscope versus Macintosh laryngoscope for vocal cords visualisation after thyroid and parathyroid surgery. Anaesth Int Care 2011; 39: 511-2.

4. Timanaykar RT, Anand LK, Palta S. A randomized controlled study to evaluate and compare TruView blade with Macintosh blade for laryngoscopy and intubation under general anesthesia. J Anaesthesiol Clin Pharmacol 2011; 27: 199-204.

5. Khan RM, Maroof M, Jain S, Khan FR, Madhu M. Truview evo-2 vs macintosh laryngoscopy: study of cardiovascular responses \& POGO scoring. J Anaesthesiol Clin Pharmacol 2008; 24: 311-4.

Nishant Kumar DNB

Debadutta Behera MD

Jaspal Singh Dali MD

Maulana Azad Medical College and Lok Nayak Hospital, New Delhi, India 